\title{
Scalar field and electromagnetic perturbations on Locally Rotationally Symmetric spacetimes
}

\author{
Gerold Betschart $\S \dagger$ and Chris A. Clarkson $\dagger \ddagger$ \\ $\dagger$ Department of Mathematics and Applied Mathematics, University of Cape \\ Town, 7701 Rondebosch, South Africa \\ $\S$ Department of Electromagnetics, Chalmers University of Technology, SE-412 \\ 96 Göteborg, Sweden \\ $\ddagger$ Institute of Cosmology and Gravitation, University of Portsmouth, \\ Portsmouth PO1 2EG, Britain \\ E-mail: geroldb@maths.uct.ac.za, chris.clarkson@port.ac.uk
}

\begin{abstract}
We study scalar field and electromagnetic perturbations on Locally Rotationally Symmetric (LRS) class II spacetimes, exploiting a recently developed covariant and gauge-invariant perturbation formalism. From the Klein-Gordon equation and Maxwell's equations, respectively, we derive covariant and gaugeinvariant wave equations for the perturbation variables and thereby find the generalised Regge-Wheeler equations for these LRS class II spacetime perturbations. As illustrative examples, the results are discussed in detail for the Schwarzschild and Vaidya spacetime, and briefly for some classes of dust Universes.

PACS numbers: 04.20.-q, 04.40.-b
\end{abstract}

\section{Introduction}

The covariant $1+3$ approach (see [1] and references therein) has proven to be a powerful tool in relativistic cosmology, especially through its application of the gauge-invariant, covariant perturbation formalism. This perturbation formalism works extremely well in cosmological applications when the background model is homogeneous and isotropic, that is of Friedman-Lemaitre-Robertson-Walker (FLRW) type. However, if the spacetime under consideration has less symmetry, the $1+3$ approach is no longer ideally adopted because its splitting in 'time' and 'space' relative to the fundamental observer is not sensitive to another preferred direction apart from 'time'. The description of space-time through covariant quantities, defined in the observer's restspace, is simply blind to a second distinguished direction. The $1+1+2$ approach [2] remedies this by slicing the 'space' further into 'sheets' orthogonal to a second preferred direction. Analogously, the quantities of the restspace are further covariantly split in such a way that obtained quantities still have a clear geometrical or physical meaning. The $1+1+2$ approach thus naturally extends the $1+3$ approach and keeps its benefits.

In this article, we illustrate the $1+1+2$ formalism with its application to scalar and electromagnetic perturbations on spacetimes which are locally rotationally symmetric (LRS) $[3,4]$ possessing a continuous isotropy group at each point and hence a multiplytransitive isometry group. Since LRS spacetimes exhibit locally a preferred spatial 
direction, the $1+1+2$ formalism is ideally suited for a covariant description of these spacetimes, yielding a complete characterisation in terms of invariant scalar quantities which have either a clear physical or direct geometrical meaning. Such a covariant classification of LRS perfect fluid spacetime geometries has already been presented in [5], whereas orthonormal frame methods have been employed in [6-8]. We will include LRS imperfect fluids in our treatment but the emphasis will be mainly on LRS class II spacetimes, that is LRS spacetimes without vorticity terms such that the sheet becomes a genuine 2 -surface.

Schwarzschild black hole perturbations are well understood and it has been known for a long time that they are all governed by master equations known as the ReggeWheeler equation [9], a Schrödinger equation with a slightly different potential for scalar, electromagnetic and gravitational perturbations, respectively [9-14], due to the differing spins of the perturbing fields. Using the $1+1+2$ formalism, we find the covariant generalisation of the Regge-Wheeler equation for scalar perturbations, as described by the Klein-Gordon equation, for all LRS spacetimes, and present the generalised Regge-Wheeler equation for electromagnetic perturbations, governed by Maxwell's equations, in the case of LRS class II spacetimes. We discuss the resulting wave equations in detail for Schwarzschild and Vaidya spacetimes [15,16], the latter being closely related to the former by having a non-increasing mass. We also describe (source-free) electromagnetic perturbations on the Schwarzschild geometry by a linear first order system of ODEs plus an algebraic constraint, once spherical and time harmonics have been introduced. This allows for a quick determination of some electromagnetic field configurations, such as the solutions describing a static magnetic dipole or a static uniform magnetic field at infinity. We also discuss the key wave equations for two classes of dust Universe models. Specifically, we have a look at the inhomogeneous Lemaitre-Tolman-Bondi (LTB) spacetimes [15, 17-19], and the spatially homogeneous Kantowski-Sachs (KS) spacetime $[15,20,21]$ in the spherical symmetric case.

\section{Preliminaries}

Let us briefly review the basics of the $1+1+2$ covariant approach and introduce the required notation, referring the reader to [2] for a more elaborate exposition. We adopt units such that $8 \pi G=1=c$ and Einstein's equations are $G_{a b}=T_{a b}-\Lambda g_{a b}$.

While the $1+3$ approach [1] provides a threading of spacetime into 'time' and 'space' with the means of a timelike unit vector field $u^{a}\left(u^{a} u_{a}=-1\right)$, representing the observers' 4 -velocity, the $1+1+2$ formalism in addition performs a slicing of the 'space' with the help of a spacelike unit vector field $n^{a}$, which is orthogonal to $u^{a}$ : $n^{a} n_{a}=1, u^{a} n_{a}=0$. The $1+3$ projection tensor $h_{a}^{b} \equiv g_{a}^{b}+u_{a} u^{b}$ combined with $n^{a}$ gives rise to a new projection tensor $N_{a b}$,

$$
N_{a}^{b} \equiv h_{a}^{b}-n_{a} n^{b}=g_{a}^{b}+u_{a} u^{b}-n_{a} n^{b},
$$

which projects vectors orthogonal to $n^{a}$ and $u^{a}\left(n^{a} N_{a b}=0=u^{a} N_{a b}\right)$ onto 2surfaces $\left(N_{a}{ }^{a}=2\right)$ which are referred to as the 'sheets'. Its volume element, the alternating Levi-Civita 2-tensor, is derived from the volume element $\varepsilon_{a b c} \equiv u^{d} \eta_{d a b c}$ for the observers' restspaces by

$$
\varepsilon_{a b} \equiv \varepsilon_{a b c} n^{c}=u^{d} \eta_{d a b c} n^{c} ; \quad \varepsilon_{a b} n^{b}=0=\varepsilon_{(a b)} .
$$

The covariant $1+3$ threading irreducibly splits any 4 -vector into a scalar part parallel to $u^{a}$ and a 3 -vector part orthogonal to $u^{a}$. Furthermore, any second rank 
tensor is covariantly and irreducibly split into scalar, 3 -vector and projected symmetric trace-free (PSTF) 3-tensor parts. The $1+1+2$ slicing takes this split further by irreducibly decomposing the 3 -vectors and PSTF 3 -tensors with respect to $n^{a}$. For example, any 3 -vector $\psi^{a}$ can now be irreducibly split into a scalar, $\Psi$, which is the part of the vector parallel to $n^{a}$, and a vector, $\Psi^{a}$, lying in the sheet orthogonal to $n^{a}$ :

$\psi^{a}=\Psi n^{a}+\Psi^{a}$, where $\Psi \equiv \psi_{a} n^{a}, \quad$ and $\quad \Psi^{a} \equiv N^{a b} \psi_{b} \equiv \psi^{\bar{a}}$,

where we use a bar over an index to denote projection with $N_{a b}$. Similarly, any PSTF tensor, $\psi_{a b}$, can now be split into scalar, vector and tensor (which are PSTF with respect to $n^{a}$ ) parts:

$$
\psi_{a b}=\psi_{\langle a b\rangle}=\Psi\left(n_{a} n_{b}-\frac{1}{2} N_{a b}\right)+2 \Psi_{(a} n_{b)}+\Psi_{a b},
$$

where

$$
\begin{aligned}
\Psi & \equiv n^{a} n^{b} \psi_{a b}=-N^{a b} \psi_{a b}, \\
\Psi_{a} & \equiv N_{a}^{b} n^{c} \psi_{b c}=\Psi_{\bar{a}}, \\
\Psi_{a b} & \equiv\left(N_{(a}^{c} N_{b)}{ }^{d}-\frac{1}{2} N_{a b} N^{c d}\right) \psi_{c d} \equiv \Psi_{\{a b\}} .
\end{aligned}
$$

We use curly brackets to denote the PSTF with respect to $n^{a}$ part of a tensor. Note that for 2nd-rank tensors in the $1+1+2$ formalism 'PSTF' is precisely equivalent to 'transverse-traceless'.

In the $1+1+2$ formalism three derivatives are used, which $u^{a}$ and $n^{a}$ define, for any object $\psi_{\ldots} \ldots$ :

$$
\begin{aligned}
\dot{\psi}_{a \cdots b}{ }^{c \cdots d} & \equiv u^{e} \nabla_{e} \psi_{a \cdots b}{ }^{c \cdots d}, \\
\hat{\psi}_{a \cdots b}{ }^{c \cdots d} & \equiv n^{e} \mathrm{D}_{e} \psi_{a \cdots b}{ }^{c \cdots d} \equiv n^{e} h_{e}{ }^{j} h_{a}{ }^{f} \cdots h_{b}{ }^{g} h_{h}{ }^{c} \cdots h_{i}{ }^{d} \nabla_{j} \psi_{f \cdots g}{ }^{h \cdots i}, \\
\delta_{e} \psi_{a \cdots b}{ }^{c \cdots d} & \equiv N_{e}{ }^{j} N_{a}{ }^{f} \cdots N_{b}{ }^{g} N_{h}{ }^{c} \cdots N_{i}{ }^{d} \mathrm{D}_{j} \psi_{f \cdots g}{ }^{h \cdots i} .
\end{aligned}
$$

The hat-derivative, analogously defined to the usual time (dot) derivative, is the derivative along the vector field $n^{a}$ in the surfaces orthogonal to $u^{a}$. The $\delta$-derivative, defined by equation (8) similarly to the spatial derivative $\mathrm{D}$, is a projected derivative on the sheet, with projection on every free index.

With these definitions the spatial derivative of $n^{a}$ orthogonal to $u^{a}$ is decomposed as follows:

$$
\mathrm{D}_{a} n_{b}=n_{a} a_{b}+\frac{1}{2} \phi N_{a b}+\xi \varepsilon_{a b}+\zeta_{a b},
$$

where

$$
\begin{aligned}
a_{a} & \equiv n^{c} \mathrm{D}_{c} n_{a}=\hat{n}_{a}, \\
\phi & \equiv \delta_{a} n^{a}, \\
\xi & \equiv \frac{1}{2} \varepsilon^{a b} \delta_{a} n_{b}, \\
\zeta_{a b} & \equiv \delta_{\{a} n_{b\}} .
\end{aligned}
$$

These have a meaning analogous to the kinematical quantities 4-acceleration $\dot{u}^{a}$, expansion $\theta$, vorticity $\omega^{a}$ and shear $\sigma_{a b}$ of the congruence $u^{a}$ in the $1+3$ formalism, which are related via

$$
\nabla_{a} u_{b}=-u_{a} \dot{u}_{b}+\frac{1}{3} \theta h_{a b}+\varepsilon_{a b c} \omega^{c}+\sigma_{a b} ;
$$

more precisely, travelling along $n^{a}, \phi$ represents the sheet expansion, $\zeta_{a b}$ is the shear of $n^{a}$ (distortion of the sheet), and $a^{a}$ its acceleration, while $\xi$ represents a 'twisting' 
of the sheet (the rotation of $n^{a}[22]$ ). The other derivative of $n^{a}$ is its change along $u^{a}$,

$$
\dot{n}_{a}=\mathcal{A} u_{a}+\alpha_{a} \quad \text { where } \quad \alpha_{a} \equiv \dot{n}_{\bar{a}} \quad \text { and } \quad \mathcal{A}=n^{a} \dot{u}_{a} .
$$

The new variables $a_{a}, \phi, \xi, \zeta_{a b}, \mathcal{A}$ and $\alpha_{a}$ are fundamental objects in the spacetime within the $1+1+2$ approach since the spacetime geometry can be inferred from them. They are treated on the same footing as the kinematical variables of $u^{a}$ in the $1+3$ approach.

The kinematical $1+3$ quantities, the Weyl curvature and the energy-momentum tensor need also to be split. For the 4 -acceleration, vorticity and shear, one arrives at

$$
\begin{aligned}
\dot{u}^{a} & =\mathcal{A} n^{a}+\mathcal{A}^{a}, \\
\omega^{a} & =\Omega n^{a}+\Omega^{a}, \\
\sigma_{a b} & =\Sigma\left(n_{a} n_{b}-\frac{1}{2} N_{a b}\right)+2 \Sigma_{(a} n_{b)}+\Sigma_{a b},
\end{aligned}
$$

while for the electric and magnetic Weyl tensors one gets

$$
\begin{aligned}
E_{a b} & =\mathcal{E}\left(n_{a} n_{b}-\frac{1}{2} N_{a b}\right)+2 \mathcal{E}_{(a} n_{b)}+\mathcal{E}_{a b}, \\
H_{a b} & =\mathcal{H}\left(n_{a} n_{b}-\frac{1}{2} N_{a b}\right)+2 \mathcal{H}_{(a} n_{b)}+\mathcal{H}_{a b} .
\end{aligned}
$$

The fluid variables heat flux $q^{a}$ and anisotropic pressure $\pi_{a b}$, inferred from the energymomentum tensor $T_{a b}$ together with the energy density $\mu$ and the isotropic pressure $p$, are split into

$$
\begin{aligned}
& q^{a}=Q n^{a}+Q^{a}, \\
& \pi_{a b}=\Pi\left(n_{a} n_{b}-\frac{1}{2} N_{a b}\right)+2 \Pi_{(a} n_{b)}+\Pi_{a b} .
\end{aligned}
$$

Finally, the covariant $1+1+2$ equations for all the above introduced quantities may be obtained from the Bianchi and Ricci identities for both the timelike unit vector field $u^{a}$ and the spacelike unit vector field $n^{a}$.

\section{LRS class II spacetimes}

In this section, we discuss LRS class II spacetimes in terms of the $1+1+2$ formalism. The discussion follows in parts van Elst \& Ellis [5] but generalises their treatment of LRS class II perfect fluids towards imperfect fluids employing a new, somewhat streamlined notation.

The $1+1+2$ formalism is ideally suited to study spacetimes which exhibit local rotational symmetry (LRS) because they have a unique preferred spatial direction at each point, defined covariantly, for example, by an eigendirection of a degenerate rate of the shear tensor field or by a vorticity vector field. This direction constitutes a local axis of symmetry - all observations are identical under rotations about it and are the same in all spatial directions perpendicular to it [3,4]. Hence, after a $1+1+2$ split, if the spacelike unit vector field $n^{a}\left(n^{a} n_{a}=1, n^{a} u_{a}=0\right)$ is chosen parallel to the axis of symmetry, all covariantly defined $1+1+2$ vectors and tensors must vanish due to

the LRS symmetry. It follows that LRS spacetimes may be covariantly characterised by scalar quantities (modulo equations of state for the matter variables), namely

$$
\text { LRS : } \quad\{\mathcal{A}, \theta, \phi, \xi, \Sigma, \Omega, \mathcal{E}, \mathcal{H}, \mu, p, Q, \Pi, \Lambda\} .
$$

The variables (23) thus fully describe LRS spacetimes and are what is solved for in the $1+1+2$ approach. For LRS class II, one requires in addition that the vorticity 
terms vanish, $\Omega=0=\xi$, which further constrains the magnetic Weyl curvature $\mathcal{H}$ to vanish. Thus, a substantially smaller set of scalars describes LRS class II spacetimes:

LRS class II : $\quad\{\mathcal{A}, \theta, \phi, \Sigma, \mathcal{E}, \mu, p, Q, \Pi, \Lambda\}$.

These LRS class II quantities satisfy a bunch of covariant evolution and/or propagation equations, obtained from the Bianchi and Ricci identities for the unit vector fields $u^{a}$ and $n^{a}$, respectively:

Propagation:

$$
\begin{array}{ll}
\hat{\phi} & =-\frac{1}{2} \phi^{2}+\left(\frac{1}{3} \theta+\Sigma\right)\left(\frac{2}{3} \theta-\Sigma\right)-\frac{2}{3}(\mu+\Lambda)-\mathcal{E}-\frac{1}{2} \Pi, \\
\hat{\Sigma}-\frac{2}{3} \hat{\theta} & =-\frac{3}{2} \phi \Sigma-Q, \\
\hat{\mathcal{E}}-\frac{1}{3} \hat{\mu}+\frac{1}{2} \hat{\Pi} & =-\frac{3}{2} \phi\left(\mathcal{E}+\frac{1}{2} \Pi\right)+\left(\frac{1}{2} \Sigma-\frac{1}{3} \theta\right) Q
\end{array}
$$

Evolution:

$\dot{\phi} \quad=-\left(\Sigma-\frac{2}{3} \theta\right)\left(\mathcal{A}-\frac{1}{2} \phi\right)+Q$,

$\dot{\Sigma}-\frac{2}{3} \dot{\theta}=-\mathcal{A} \phi+2\left(\frac{1}{3} \theta-\frac{1}{2} \Sigma\right)^{2}+\frac{1}{3}(\mu+3 p-2 \Lambda)-\mathcal{E}+\frac{1}{2} \Pi$,

$\dot{\mathcal{E}}-\frac{1}{3} \dot{\mu}+\frac{1}{2} \dot{\Pi}=+\left(\frac{3}{2} \Sigma-\theta\right) \mathcal{E}+\frac{1}{4}\left(\Sigma-\frac{2}{3} \theta\right) \Pi+\frac{1}{2} \phi Q-\frac{1}{2}(\mu+p)\left(\Sigma-\frac{2}{3} \theta\right) ;$

Propagation/evolution:

$$
\begin{aligned}
& \hat{\mathcal{A}}-\dot{\theta}=-(\mathcal{A}+\phi) \mathcal{A}+\frac{1}{3} \theta^{2}+\frac{3}{2} \Sigma^{2}+\frac{1}{2}(\mu+3 p-2 \Lambda), \\
& \dot{\mu}+\hat{Q}=-\theta(\mu+p)-(\phi+2 \mathcal{A}) Q-\frac{3}{2} \Sigma \Pi, \\
& \dot{Q}+\hat{p}+\hat{\Pi}=-\left(\frac{3}{2} \phi+\mathcal{A}\right) \Pi-\left(\frac{4}{3} \theta+\Sigma\right) Q-(\mu+p) \mathcal{A} .
\end{aligned}
$$

This system of equations extends the corresponding system in [5] (see section 6) to imperfect fluids.

Since the vorticity $\Omega$ vanishes, the unit vector field $u^{a}$ is hypersurface-orthogonal to the spacelike 3 -surfaces whose intrinsic curvature can be calculated from the Gauss equation for $u^{a}$. For the intrinsic Ricci-curvature, one finds from this

$$
\begin{aligned}
{ }^{3} R_{a b}= & {\left[\frac{2}{3}(\mu+\Lambda)+\mathcal{E}+\frac{1}{2} \Pi+\Sigma^{2}-\frac{1}{3} \theta \Sigma-\frac{2}{9} \theta^{2}\right] n_{a} n_{b} } \\
& +\left[\frac{2}{3}(\mu+\Lambda)-\frac{1}{2} \mathcal{E}-\frac{1}{4} \Pi+\frac{1}{4} \Sigma^{2}+\frac{1}{6} \theta \Sigma-\frac{2}{9} \theta^{2}\right] N_{a b} .
\end{aligned}
$$

The intrinsic Ricci-scalar of the 3 -surfaces is therefore

$$
{ }^{3} R=2\left[\mu+\Lambda-\frac{1}{3} \theta^{2}+\frac{3}{4} \Sigma^{2}\right] .
$$

This relation constitutes the generalised Friedman equation.

On the other hand, the additional vanishing of the sheet distortion, $\xi$, implies that the sheet is in this case a genuine 2 -surface. The Gauss equation for $n^{a}$ together with the 3-Ricci identities determine the 3-Ricci curvature tensor of the spacelike 3-surfaces orthogonal to $u^{a}$ to be

$$
{ }^{3} R_{a b}=-\left[\hat{\phi}+\frac{1}{2} \phi^{2}\right] n_{a} n_{b}-\left[\frac{1}{2} \hat{\phi}+\frac{1}{2} \phi^{2}-K\right] N_{a b},
$$

thus giving for the 3 -Ricci scalar

$$
{ }^{3} R=-2\left[\hat{\phi}+\frac{3}{4} \phi^{2}-K\right] .
$$

Here, $K$ is the Gaussian curvature of the sheet, ${ }^{2} R_{a b}=K N_{a b}$. Combining the last equation with equations (35) and (25), we can express the Gaussian curvature $K$ in the form

$$
K=\frac{1}{3}(\mu+\Lambda)-\mathcal{E}-\frac{1}{2} \Pi+\frac{1}{4} \phi^{2}-\left(\frac{1}{3} \theta-\frac{1}{2} \Sigma\right)^{2} .
$$


Evolution and propagation equations for $K$ can be calculated from equations (25)-(30) to give

$$
\begin{aligned}
\dot{K} & =-\left(\frac{2}{3} \theta-\Sigma\right) K, \\
\hat{K} & =-\phi K .
\end{aligned}
$$

Every scalar $\psi$ in LRS II has to satisfy the commutation relation

$$
\hat{\dot{\psi}}-\dot{\hat{\psi}}=-\mathcal{A} \dot{\psi}+\left(\frac{1}{3} \theta+\Sigma\right) \hat{\psi} .
$$

We applied this commutator relation to the Gaussian curvature $K$ in order to check for consistency of the equations (25)-(33), which is indeed guaranteed due to equations (39) and (40).

From equation (39) follows the neat result that whenever the Gaussian curvature $K$ of the sheet is constant in time but non-vanishing, that is, the sheets have either spherical or hyperbolic geometry, the shear $\Sigma$ is proportional to the expansion $\theta$ :

$$
K \neq 0 \quad \text { and } \quad \dot{K}=0 \quad \Longrightarrow \quad \Sigma=\frac{2}{3} \theta \text {. }
$$

As a matter of frame choice, it is always possible to pick an observer with $\dot{K}=$ 0 , corresponding to a 'static' observer. Adopting such a choice leads to great simplifications in the equations, the caveat being that such a choice is often not the most natural one, e.g., in Friedman-Lemaitre-Robertson-Walker (FLRW) spacetimes. For example, taking a static observer to describe spherically symmetric spacetimes, the constraint (38) allows for a covariant definition of the radial parameter $r$ via

$$
r^{-2}=\frac{1}{3}(\mu+\Lambda)-\mathcal{E}-\frac{1}{2} \Pi+\frac{1}{4} \phi^{2} ; \quad \dot{r}=0=\delta_{a} r .
$$

(A non-static observer has $\dot{r} \neq 0$ ). In situations with spherical symmetry it is convenient to express the hat-derivative in terms of the radial parameter $r$. If we associate an affine parameter $\rho$ with the hat-derivative, we find using equation (40)

$$
\hat{X}=\frac{d X}{d \rho}=\frac{1}{2} r \phi \frac{\partial X}{\partial r}+\hat{\tau} \dot{X},
$$

where $\tau$ denotes proper time and $X$ may be any scalar.

In summary, we have found that in the $1+1+2$ formalism, all LRS II spacetimes are covariantly described by the scalar equations (25)- (33). In this case, the sheets are genuine 2-surfaces whose extrinsic geometry is determined by the Gaussian curvature $K$, which can be computed from (38). Furthermore, due to (42), we stress that static spacetimes whose sheet is either spherical or hyperbolic must have their shear $\Sigma$ proportional to the expansion $\theta$; more precisely, such imperfect fluid spacetimes fulfill the relation $\Sigma-\frac{2}{3} \theta=0$, generalising the well-known fact that perfect fluid spacetimes within this subclass possess vanishing shear $\Sigma$ and expansion $\theta$.

\section{Perturbations on LRS class II backgrounds}

In this section, we investigate scalar and electromagnetic perturbations on LRS class II background spacetimes. The scalar end electromagnetic fields are treated as test fields, i.e., they do not affect the geometry of the background. Hence, the Stewart-Walker Lemma [23] ensures that these fields are gauge-invariant. Our goal is the derivation of the concomitant covariant and gauge-invariant wave equations which describe these perturbations. These wave equations are the generalisation of the well-known ReggeWheeler equations from the Schwarzschild case towards LRS class II spacetimes, yet written in covariant notation. 


\subsection{Commutation relations}

In order to achieve this aim, we will make frequent use of commutation relations. All first order scalars $\psi$ have to satisfy the following commutation relations between the different derivatives introduced above:

$$
\begin{array}{ll}
\hat{\dot{\psi}}-\dot{\hat{\psi}} & =-\mathcal{A} \dot{\psi}+\left(\frac{1}{3} \theta+\Sigma\right) \hat{\psi}, \\
\delta_{a} \dot{\psi}-N_{a}^{b}\left(\delta_{b} \psi\right)^{\cdot} & =-\frac{1}{2}\left(\Sigma-\frac{2}{3} \theta\right) \delta_{a} \psi, \\
\delta_{a} \hat{\psi}-N_{a}^{b}\left(\delta_{b} \psi\right) & =+\frac{1}{2} \phi \delta_{a} \psi, \\
\delta_{[a} \delta_{b]} \psi & =0 .
\end{array}
$$

Analogously, the commutation relations for first order 2-vectors $\psi_{a}$ read

$$
\begin{array}{ll}
\hat{\dot{\psi}}_{\bar{a}}-\dot{\hat{\psi}}_{\bar{a}} & =-\mathcal{A} \dot{\psi}_{\bar{a}}+\left(\frac{1}{3} \theta+\Sigma\right) \hat{\psi}_{\bar{a}}, \\
\delta_{a} \dot{\psi}_{b}-N_{a}^{c} N_{b}{ }^{d}\left(\delta_{c} \psi_{d}\right) & =-\frac{1}{2}\left(\Sigma-\frac{2}{3} \theta\right) \delta_{a} \psi_{b}, \\
\delta_{a} \hat{\psi}_{b}-N_{a}^{c} N_{b}{ }^{d}\left(\delta_{c} \psi_{d}\right) & =+\frac{1}{2} \phi \delta_{a} \psi_{b}, \\
\delta_{[a} \delta_{b]} \psi_{c} & =-K \psi_{[a} N_{b] c} .
\end{array}
$$

\subsection{Harmonics}

It is useful to expand all first-order perturbations in harmonics. Note that all functions and relations below are defined in the background only; we solely expand first-order variables, so zeroth-order equations are sufficient.

In analogy to the spatial harmonics defined in [24], we introduce dimensionless sheet harmonic functions $Q$, defined on the background, as eigenfunctions of the 2dimensional Laplace-Beltrami operator such that for positive, negative or vanishing curvature $K$

$$
\delta^{2} Q=-\frac{k^{2}}{r^{2}} Q, \quad \hat{Q}=0=\dot{Q} \quad\left(0 \leq k^{2} \in \mathbb{R}\right) .
$$

The function $r$ is, up to an irrelevant constant, covariantly defined by

$$
\frac{\hat{r}}{r} \equiv \frac{1}{2} \phi, \quad \frac{\dot{r}}{r} \equiv \frac{1}{3} \theta-\frac{1}{2} \Sigma, \quad \delta_{a} r \equiv 0,
$$

which, in the light of equations (39)-(40), is nothing but the covariant version of the common setting $K \equiv \kappa / r^{2}$ with $\kappa= \pm 1$ for spherical and hyperbolic 2-geometry, respectively, where the constant is fixed by the relation (38). However, the covariant definition (54) extends to the plane-symmetric case as well, where there is no natural length scale. We can now expand any first order scalar $\psi$ in terms of these functions formally as

$$
\psi=\sum_{k} \psi_{\mathrm{S}}^{(k)} Q^{(k)}=\psi_{\mathrm{S}} Q
$$

where the sum over $k$ is implicit in the last equality. The $S$ subscript reminds us that $\psi$ is a scalar, and that a harmonic expansion has been made.

We also need to expand vectors in harmonics. We therefore define the even (electric) parity vector harmonics as

$Q_{a}^{(k)}=r \delta_{a} Q^{(k)} \quad \Rightarrow \quad \hat{Q}_{\bar{a}}=0=\dot{Q}_{\bar{a}}, \quad \delta^{2} Q_{a}=\left(1-k^{2}\right) r^{-2} Q_{a} ;$ 
where the $(k)$ superscript is implicit, and we define odd (magnetic) parity vector harmonics as

$\bar{Q}_{a}^{(k)}=r \varepsilon_{a b} \delta^{b} Q^{(k)} \quad \Rightarrow \quad \hat{\bar{Q}}_{\bar{a}}=0=\dot{\bar{Q}}_{\bar{a}}, \quad \delta^{2} \bar{Q}_{a}=\left(1-k^{2}\right) r^{-2} \bar{Q}_{a}$.

Note that $\bar{Q}_{a}=\varepsilon_{a b} Q^{b} \Leftrightarrow Q_{a}=-\varepsilon_{a b} \bar{Q}^{b}$, so that $\varepsilon_{a b}$ is a parity operator. The crucial difference between these two types of vector harmonics is that $\bar{Q}_{a}$ is solenoidal, so

$$
\delta^{a} \bar{Q}_{a}=0,
$$

while

$$
\delta^{a} Q_{a}=-k^{2} r^{-1} Q .
$$

Note also that

$$
\varepsilon_{a b} \delta^{a} Q^{b}=0, \quad \text { and } \quad \varepsilon_{a b} \delta^{a} \bar{Q}^{b}=+k^{2} r^{-1} Q .
$$

The harmonics are orthogonal: $Q^{a} \bar{Q}_{a}=0$ (for each $k$ ), which implies that any firstorder vector $\psi_{a}$ can now be written

$$
\psi_{a}=\sum_{k} \psi_{\vee}^{(k)} Q_{a}^{(k)}+\bar{\psi}_{\vee}^{(k)} \bar{Q}_{a}^{(k)}=\psi_{\vee} Q_{a}+\bar{\psi}_{\vee} \bar{Q}_{a} .
$$

Again, we implicitly assume a sum over $k$ in the last equality, and the $\mathrm{V}$ subscript reminds us that $\psi_{a}$ is a vector expanded in harmonics.

We like to point out that the harmonics introduced here naturally generalise the spherical harmonics used in [2]. In particular, the various formulae for scalar and vector spherical harmonics stated in [2] also hold for our generalised harmonics.

\subsection{Scalar perturbations}

Let us consider perturbations of LRS spacetimes due to a massive scalar field, $\psi$, with mass $M$, whose equation of motion is the Klein-Gordon equation,

$$
\left(g^{a b} \nabla_{a} \nabla_{b}+M^{2}\right) \psi=\left(\nabla^{a} \nabla_{a}+M^{2}\right) \psi=0 .
$$

We investigate this simple equation first in an arbitrary spacetime and specialising to LRS II spacetime afterwards. The corresponding $1+3$ equation is easily derived using $g^{a b}=h^{a b}-u^{a} u^{b}$ and the following expression for the spatial Laplacian of a scalar field,

$$
\mathrm{D}^{a} \mathrm{D}_{a} \psi=h^{a b} \mathrm{D}_{a} \mathrm{D}_{b} \psi=h^{a b} \nabla_{a} \nabla_{b} \psi+\theta \dot{\psi},
$$

and leads to the wave equation

$$
\ddot{\psi}-\mathrm{D}^{a} \mathrm{D}_{a} \psi+\theta \dot{\psi}-\dot{u}^{a} \mathrm{D}_{a} \psi+M^{2} \psi=0 .
$$

Thus, for a general spacetime, the evolution of a scalar field is affected by effects of expansion and acceleration, as measured by the fundamental observer.

The $1+1+2$ form of equation (64) is readily obtained using $h^{a b}=N^{a b}+n^{a} n^{b}$ as well as the relation

$$
\delta^{a} \delta_{a} \psi=N^{a b} \delta_{a} \delta_{b} \psi=N^{a b} \mathrm{D}_{a} \mathrm{D}_{b} \psi-\phi \hat{\psi} .
$$

The resulting equation reads as

$$
\ddot{\psi}-\hat{\hat{\psi}}+\theta \dot{\psi}-(\mathcal{A}+\phi) \hat{\psi}+\left(a^{a}-\mathcal{A}^{a}-\delta^{a}\right) \delta_{a} \psi+M^{2} \psi=0 .
$$


Equation (66) is the fully split Klein-Gordon equation for a massive scalar field in an arbitrary spacetime, given in a covariant and gauge-invariant fashion. For LRS spacetimes, the wave equation (66) closes, yielding

$$
\ddot{\psi}-\hat{\hat{\psi}}+\theta \dot{\psi}-(\mathcal{A}+\phi) \hat{\psi}+\left(M^{2}-\delta^{2}\right) \psi=0 .
$$

We emphasise that this is true for all LRS spacetimes. Equation (67) constitutes the generalised Regge-Wheeler equation for scalar perturbations on all LRS background spacetimes. While the generalised Regge-Wheeler equation (67) is not affected by rotational effects at all, it is in general sensitive to the observer's acceleration $\mathcal{A}$, the spacetime expansion $\theta$, the sheet expansion $\phi$, as well as the mass $M$ of the perturbation field $\psi$.

For LRS class II spacetimes, it is sometimes convenient to rescale the scalar field $\psi$ according to $\psi \equiv r^{-1} \Psi$, where the function $r$ is defined via (54). In terms of the rescaled field $\Psi$, the Regge-Wheeler equation (67) reads

$\ddot{\Psi}-\hat{\hat{\Psi}}+\left(\Sigma+\frac{1}{3} \theta\right) \dot{\Psi}-\mathcal{A} \hat{\Psi}+\left[M^{2}-\mathcal{E}-\frac{1}{6}(\mu-3 p+4 \Lambda)-\delta^{2}\right] \Psi=0$.

This form of the Regge-Wheeler equation has several advantages over (67): firstly, it allows to introduce the Gaussian curvature $K$ at the expense of the Weyl curvature $\mathcal{E}$, for example, and secondly, it simplifies in vacuo. We emphasise that this form is generic: we will show in the next section that electromagnetic perturbations are described covariantly by the same equation but having a different potential [that is, the term in square brackets in [68] ] once appropriate harmonics have been used to get rid of the sheet Laplacian $\delta^{2}$.

\subsection{Electromagnetic perturbations}

In accordance with (3), the electric, magnetic and current 3-vector fields are irreducibly decomposed into scalar and 2-vector parts as

$$
E^{a}=\mathscr{E} n^{a}+\mathscr{E}^{a}, \quad B^{a}=\mathscr{B} n^{a}+\mathscr{B}^{a}, \quad J^{a}=\mathscr{J} n^{a}+\mathscr{J}^{a} .
$$

Thus, Maxwell's equations for electromagnetic test fields, sourced by the total electric charge $\rho_{\mathrm{e}}$ and the currents $\mathscr{J}$ and $\mathscr{J}_{a}$, respectively, on a LRS class II geometry become in the sheet approach (the splitting for arbitrary spacetimes is displayed in [25]):

$$
\begin{array}{ll}
\hat{\mathscr{E}}+\delta_{a} \mathscr{E}^{a} & =-\phi \mathscr{E}+\rho_{\mathrm{e}} \\
\hat{\mathscr{B}}+\delta_{a} \mathscr{B}^{a} & =-\phi \mathscr{B} \\
\dot{\mathscr{E}}-\varepsilon_{a b} \delta^{a} \mathscr{B}^{b} & =+\left(\Sigma-\frac{2}{3} \theta\right) \mathscr{E}-\mathscr{J} \\
\dot{\mathscr{B}}+\varepsilon_{a b} \delta^{a} \mathscr{E}^{b} & =+\left(\Sigma-\frac{2}{3} \theta\right) \mathscr{E} \\
\dot{\mathscr{E}}_{\bar{a}}+\varepsilon_{a b}\left(\hat{\mathscr{B}}^{b}-\delta^{b} \mathscr{B}\right) & =-\left(\frac{1}{2} \phi+\mathcal{A}\right) \varepsilon_{a b} \mathscr{B}^{b}-\left(\frac{1}{2} \Sigma+\frac{2}{3} \theta\right) \mathscr{E}_{a}-\mathscr{J}_{a} \\
\dot{\mathscr{B}}_{\bar{a}}-\varepsilon_{a b}\left(\hat{\mathscr{E}}^{b}-\delta^{b} \mathscr{E}\right) & =+\left(\frac{1}{2} \phi+\mathcal{A}\right) \varepsilon_{a b} \mathscr{E}^{b}-\left(\frac{1}{2} \Sigma+\frac{2}{3} \theta\right) \mathscr{B}_{a} .
\end{array}
$$

Note that $\varepsilon_{a b}$ is a parity operator, $\varepsilon_{a c} \varepsilon_{b}^{c}=-N_{a b}$, thus we can switch parity between 2 -vectors $X_{a}$ and $Y_{a}$ via

$$
X_{a}=\varepsilon_{a b} Y^{b} \Longleftrightarrow Y_{a}=-\varepsilon_{a b} X^{b} .
$$

Using this relation, the parity-reversed form of equations (74) and (75) read

$$
\begin{aligned}
& \hat{\mathscr{E}}_{\bar{a}}+\varepsilon_{a b} \dot{\mathscr{B}}^{b}-\delta_{a} \mathscr{E}=-\left(\frac{1}{2} \phi+\mathcal{A}\right) \mathscr{E}_{a}-\left(\frac{1}{2} \Sigma+\frac{2}{3} \theta\right) \varepsilon_{a b} \mathscr{B}^{b} \\
& \hat{\mathscr{B}}_{\bar{a}}-\varepsilon_{a b} \dot{\mathscr{E}}^{b}-\delta_{a} \mathscr{B}=+\left(\frac{1}{2} \phi+\mathcal{A}\right) \mathscr{B}_{a}-\left(\frac{1}{2} \Sigma+\frac{2}{3} \theta\right) \varepsilon_{a b} \mathscr{E}^{b}+\varepsilon_{a b} \mathscr{J}^{b} .
\end{aligned}
$$


From Maxwell's equations, one deduces (by a somewhat tedious calculation exploiting the earlier displayed commutation relations as well as the above parity-reversed equations) the following wave equations for the electromagnetic fields along the distinguished direction $n_{a}$ :

$$
\begin{aligned}
\ddot{\mathscr{E}} & -\hat{\hat{\mathscr{E}}}-(\mathcal{A}+2 \phi) \hat{\mathscr{E}}-\left(\Sigma-\frac{5}{3} \theta\right) \dot{\mathscr{E}} \\
& -\left[\delta^{2}+\frac{1}{2} \phi^{2}-2 \mathcal{E}+\left(\frac{1}{3} \theta+\Sigma\right)\left(\frac{3}{2} \Sigma-\theta\right)-\frac{1}{3}(\mu-3 p+4 \Lambda)\right] \mathscr{E} \\
& =\hat{\rho}_{\mathrm{e}}+\dot{\mathscr{J}}+(\phi+\mathcal{A}) \rho_{\mathrm{e}}+\theta \mathscr{J}, \\
\ddot{\mathscr{B}}- & \hat{\hat{\mathscr{B}}}-(\mathcal{A}+2 \phi) \hat{\mathscr{B}}-\left(\Sigma-\frac{5}{3} \theta\right) \dot{\mathscr{B}} \\
& -\left[\delta^{2}+\frac{1}{2} \phi^{2}-2 \mathcal{E}+\left(\frac{1}{3} \theta+\Sigma\right)\left(\frac{3}{2} \Sigma-\theta\right)-\frac{1}{3}(\mu-3 p+4 \Lambda)\right] \mathscr{B} \\
& =0 .
\end{aligned}
$$

Equations (79)- 800 are the generalisation of the famous Regge-Wheeler equation, for electromagnetic perturbations on the Schwarzschild background, towards LRS class II spacetimes, although written in covariant manner. We emphasise that $\mathscr{E}$ and $\mathscr{B}$ decouple from each other and that in the absence of sources the equations are identical closed wave equations.

It will turn out advantageous to rescale the fields and the sources under consideration similarly as above for Klein-Gordon fields; that is, we define

$$
\mathscr{E} \equiv r^{-2} E, \quad \mathscr{B} \equiv r^{-2} B, \quad \rho_{\mathrm{e}} \equiv r^{-2} \varrho_{\mathrm{e}}, \quad \mathscr{J} \equiv r^{-2} J,
$$

and substitute into the wave equations (79) (80) to obtain

$$
\begin{aligned}
\ddot{E} & -\hat{\hat{E}}-\mathcal{A} \hat{E}+\left(\Sigma+\frac{1}{3} \theta\right) \dot{E}-\left[\delta^{2}+\left(\Sigma-\frac{2}{3} \theta\right)\left(2 \Sigma+\frac{1}{6} \theta\right)\right] E \\
& =\hat{\varrho}_{\mathrm{e}}+\dot{J}+\mathcal{A} \varrho_{\mathrm{e}}+\left(\Sigma+\frac{1}{3} \theta\right) J \\
\ddot{B} & -\hat{\hat{B}}-\mathcal{A} \hat{B}+\left(\Sigma+\frac{1}{3} \theta\right) \dot{B}-\left[\delta^{2}+\left(\Sigma-\frac{2}{3} \theta\right)\left(2 \Sigma+\frac{1}{6} \theta\right)\right] B=0 .
\end{aligned}
$$

Again, if we neglect the source terms, the equations become identical closed wave equations. Moreover, the equations (82)- (83) are of the same form as equation (68) for the Klein-Gordon field, the only difference being a slightly altered potential term.

The wave equations for the electromagnetic perturbations lying in the sheet are derived analogously and read

$$
\begin{aligned}
\ddot{\mathscr{E}}_{\bar{a}}-\hat{\hat{\mathscr{E}}}_{\bar{a}}- & (3 \mathcal{A}+\phi) \hat{\mathscr{E}}_{\bar{a}}-\left(\Sigma-\frac{5}{3} \theta\right) \dot{\mathscr{E}}_{\bar{a}} \\
+ & {\left[\frac{1}{4} \phi^{2}-\mathcal{E}-\mathcal{A}(\mathcal{A}+\phi)+\frac{2}{9} \theta^{2}-\frac{2}{3} \theta \Sigma-\frac{7}{4} \Sigma^{2}+\frac{1}{3}(\mu-3 p+4 \Lambda)-\delta^{2}\right] \mathscr{E}_{a} } \\
= & (\phi-2 \mathcal{A}) \delta_{a} \mathscr{E}+3 \Sigma \varepsilon_{a b} \hat{\mathscr{B}}^{b}+(3 \mathcal{A} \Sigma-Q+\hat{\theta}-\dot{\mathcal{A}}) \varepsilon_{a b} \mathscr{B}^{b} \\
& -\delta_{a} \rho_{\mathrm{e}}-\dot{\mathscr{J}}_{\bar{a}}-\left(\theta-\frac{3}{2} \Sigma\right) \mathscr{J}_{a} \\
\ddot{\mathscr{B}}_{\bar{a}}-\hat{\hat{\mathscr{B}}}_{\bar{a}}- & (3 \mathcal{A}+\phi) \hat{\mathscr{B}}_{\bar{a}}-\left(\Sigma-\frac{5}{3} \theta\right) \dot{\mathscr{B}}_{\bar{a}} \\
+ & {\left[\frac{1}{4} \phi^{2}-\mathcal{E}-\mathcal{A}(\mathcal{A}+\phi)+\frac{2}{9} \theta^{2}-\frac{2}{3} \theta \Sigma-\frac{7}{4} \Sigma^{2}+\frac{1}{3}(\mu-3 p+4 \Lambda)-\delta^{2}\right] \mathscr{B}_{a} } \\
= & (\phi-2 \mathcal{A}) \delta_{a} \mathscr{B}-3 \Sigma \varepsilon_{a b} \hat{\mathscr{E} b}-(3 \mathcal{A} \Sigma-Q+\hat{\theta}-\dot{\mathcal{A}}) \varepsilon_{a b} \mathscr{E}^{b} \\
& +\varepsilon_{a b}\left[\delta^{b} \mathscr{J}-\hat{\mathscr{J}}^{b}-\left(\frac{1}{2} \phi+2 \mathcal{A}\right) \mathscr{J}^{b}\right] .
\end{aligned}
$$

In contrast to equations (79)sources. For example, in addition to the source terms, the magnetic 2 -vector field $\mathscr{B}_{a}$ 
gives rise to forcing terms for the electric 2 -vector field $\mathscr{E}_{a}$, which is also forced by the 'radial' electric field.

However, it turns out that in the absence of sources knowledge of the 'radial' part $\mathscr{E}$ (or $\mathscr{B}$ ) of the perturbations, e.g., by solving the concomitant Regge-Wheeler equation (82), suffices to completely determine the electromagnetic perturbations. To see this, we expand all perturbations into harmonics and decompose the governing Maxwell's equations into their harmonic components. (For details, we refer the reader to Ref. [2], Section E). From the scalar equations (70)-(73) we find for each fixed harmonic index $k$

$$
\begin{array}{ll}
\hat{\mathscr{E}}_{\mathrm{S}}+\phi \mathscr{E}_{\mathrm{S}} & =+\frac{k^{2}}{r} \mathscr{E}_{\mathrm{V}}, \\
\hat{\mathscr{B}}_{\mathrm{S}}+\phi \mathscr{B}_{\mathrm{S}} & =+\frac{k^{2}}{r} \mathscr{B}_{\mathrm{V}}, \\
\dot{\mathscr{E}}_{\mathrm{S}}-\left(\Sigma-\frac{2}{3} \theta\right) \mathscr{E}_{\mathrm{S}} & =+\frac{k^{2}}{r} \overline{\mathscr{B}}_{\mathrm{V}}, \\
\dot{\mathscr{B}}_{\mathrm{S}}-\left(\Sigma-\frac{2}{3} \theta\right) \mathscr{B}_{\mathrm{S}} & =-\frac{k^{2}}{r} \overline{\mathscr{E}}_{\mathrm{V}} .
\end{array}
$$

It is thus obvious that a solution for the 'radial' fields $\mathscr{E}$ and $\mathscr{B}$ determines the sheet fields $\mathscr{E}_{a}$ and $\mathscr{B}_{a}$, respectively.

The 2-vector equations (74) and (75), or (77) and (78), respectively, split into two parts; the first part is the odd parity part, given by

$$
\begin{aligned}
& \dot{\overline{\mathscr{E}}}_{\mathrm{V}}+\hat{\mathscr{B}}_{\mathrm{V}}-\frac{1}{r} \mathscr{B}_{\mathrm{S}}=-\left(\frac{1}{2} \phi+\mathcal{A}\right) \mathscr{B}_{\mathrm{V}}-\left(\frac{1}{2} \Sigma+\frac{2}{3} \theta\right) \overline{\mathscr{E}}_{\mathrm{V}} \\
& \dot{\overline{\mathscr{B}}}_{\mathrm{V}}-\hat{\mathscr{E}}_{\mathrm{V}}+\frac{1}{r} \mathscr{E}_{\mathrm{S}}=+\left(\frac{1}{2} \phi+\mathcal{A}\right) \mathscr{E} \mathrm{V}-\left(\frac{1}{2} \Sigma+\frac{2}{3} \theta\right) \overline{\mathscr{B}}_{\mathrm{V}}
\end{aligned}
$$

and the second part is the even parity part, given by

$$
\begin{aligned}
& \dot{\mathscr{E}}_{\mathrm{V}}-\hat{\overline{\mathscr{B}}}_{\mathrm{V}}=+\left(\frac{1}{2} \phi+\mathcal{A}\right) \overline{\mathscr{B}}_{\mathrm{V}}-\left(\frac{1}{2} \Sigma+\frac{2}{3} \theta\right) \mathscr{E} \mathrm{V}, \\
& \dot{\mathscr{B}}_{\mathrm{V}}+\hat{\overline{\mathscr{E}}}_{\mathrm{V}}=-\left(\frac{1}{2} \phi+\mathcal{A}\right) \overline{\mathscr{E}}_{\mathrm{V}}-\left(\frac{1}{2} \Sigma+\frac{2}{3} \theta\right) \mathscr{B} \mathrm{V} .
\end{aligned}
$$

We remark that the even parity equations are redundant since propagating the constraints (88)-(89) and inserting them into (92) and (93) just yields back equation (86) and (87). On the other hand, the odd parity equations are not implied by the scalar ones and have been used in the derivation of the generalised ReggeWheeler equation (79). If one eliminates $\overline{\mathscr{E}}_{V}$ and $\overline{\mathscr{B}}_{V}$ from equations (92)-(93) using the constraints (88)- (89), it becomes obvious that the electromagnetic perturbations fall into two distinct classes whose equations decouple from each other, namely:

$$
\begin{array}{ll}
\text { polar perturbations : } & \left\{\mathscr{E}_{\mathrm{S}}, \mathscr{E}_{\mathrm{V}}, \overline{\mathscr{B}}_{\mathrm{V}}\right\}, \\
\text { axial perturbations : } & \left\{\mathscr{B}_{\mathrm{S}}, \mathscr{B}_{\mathrm{V}}, \overline{\mathscr{E}}_{\mathrm{V}}\right\} .
\end{array}
$$

Moreover, the resulting equations involving either $\mathscr{E}_{S}$ and $\mathscr{E} V$ or $\mathscr{B}_{S}$ and $\mathscr{B}$ V are identical.

\section{Examples}

We have shown that scalar and vector perturbations in LRS class II spacetimes are governed by simple master equations. We shall now discuss these master equations in some specific spacetimes. First, we shall recover the well known Regge-Wheeler equations for the Schwarzschild spacetime, which we then generalise to the Vaidya radiation spacetime. We then discuss solutions from the Lemaitre-Tolman-Bondi and Kantowski-Sachs families. 


\subsection{The Schwarzschild spacetime}

5.1.1. The background Schwarzschild spacetime is fully determined by any two of three non-zero scalar functions $\phi, \mathcal{A}, \mathcal{E}$. These functions obey the background equations

$$
\begin{aligned}
& \hat{\phi}=-\frac{1}{2} \phi^{2}+\mathcal{A} \phi, \\
& \hat{\mathcal{A}}=-\mathcal{A}(\phi+\mathcal{A}) ;
\end{aligned}
$$

together with the constraint

$$
\mathcal{E}+\mathcal{A} \phi=0 .
$$

The parametric solutions for these variables are [2]

$$
\begin{aligned}
\mathcal{E} & =-\frac{2 m}{r^{3}}, \\
\phi & =\frac{2}{r} \sqrt{1-\frac{2 m}{r}}, \\
\mathcal{A} & =\frac{m}{r^{2}}\left(1-\frac{2 m}{r}\right)^{-1 / 2} .
\end{aligned}
$$

These form a one-parameter family of solutions, parameterised by the constant $m$, which is just the Schwarzschild mass. The (exterior) Schwarzschild solution is given for $2 m<r<\infty$.

5.1.2. The perturbations In order to make the connection with the standard ReggeWheeler equations [13], it is instructive to contrast the found covariant and gaugeinvariant wave equations for scalar and electromagnetic perturbations with the ones governing gravitational perturbations. The fundamental object for the study of gravitational perturbations on a Schwarzschild background is the Regge-Wheeler tensor $W_{a b}[2]$, defined by

$$
W_{a b}=\frac{1}{2} \phi r^{2} \zeta_{a b}-\frac{1}{3} r^{2} \mathcal{E}^{-1} \delta_{\{a} \delta_{b\}} \mathcal{E},
$$

a dimensionless, gauge-invariant, transverse-traceless tensor which obeys the wave equation

$$
\ddot{W}_{\{a b\}}-\hat{\hat{W}}_{\{a b\}}-\mathcal{A} \hat{W}_{\{a b\}}+\phi^{2} W_{a b}-\delta^{2} W_{a b}=0 .
$$

If one expands equation (103) into spherical tensor harmonics, the odd and even parity parts of equation (103) both become

$$
\ddot{W}_{\mathrm{T}}-\hat{\hat{W}}_{\mathrm{T}}-\mathcal{A} \hat{W}_{\mathrm{T}}+\left[\frac{L}{r^{2}}+3 \mathcal{E}\right] W_{\mathrm{T}}=0,
$$

where $W_{\mathrm{T}}=W_{\mathrm{T}}^{(\ell)}$ are the tensor harmonic components of $W_{a b}, \ell=1,2, \ldots$ and $L=\ell(\ell+1)=k^{2}$. It turns out that equation (104) is actually the Regge-Wheeler equation [9] when written in appropriate coordinates. Converting from $\rho$, the affine parameter associated with the hat-derivative, to the parameter $r, \rho \rightarrow r$, and then to the 'tortoise' coordinate of Regge and Wheeler,

$$
r_{*}=r+2 m \ln \left(\frac{r}{2 m}-1\right)
$$


and also introducing the Schwarzschild time via $d \tau=\sqrt{1-\frac{2 m}{r}} d t$, we find that (104) becomes:

$$
\left(-\frac{\partial^{2}}{\partial t^{2}}+\frac{\partial^{2}}{\partial r_{*}^{2}}+\mathscr{V}_{T}\right) W_{\mathrm{T}}=0, \quad \mathscr{V}_{T}=\left(1-\frac{2 m}{r}\right)\left[\frac{L}{r^{2}}-\frac{6 m}{r^{3}}\right],
$$

where $\mathscr{V}_{T}$ is the Regge-Wheeler potential for gravitational perturbations.

It is now straightforward to obtain the familiar Regge-Wheeler equations for the case of scalar and electromagnetic perturbations. From equation (68) one finds immediately for a massive scalar field $\psi$ with mass $M, \psi=r^{-1} \Psi$, that

$$
\ddot{\Psi}-\hat{\hat{\Psi}}-\mathcal{A} \hat{\Psi}-\left[\mathcal{E}-M^{2}+\delta^{2}\right] \Psi=0 \text {. }
$$

Introducing scalar spherical harmonics and performing as above one readily gets

$$
\left(-\frac{\partial^{2}}{\partial t^{2}}+\frac{\partial^{2}}{\partial r_{*}^{2}}+\mathscr{V}_{S}\right) \Psi_{\mathrm{S}}=0, \quad \mathscr{V}_{S}=\left(1-\frac{2 m}{r}\right)\left[\frac{L}{r^{2}}+\frac{2 m}{r^{3}}+M^{2}\right],
$$

where $\Psi_{\mathrm{S}}$ is a scalar harmonic component of $\Psi$ and $\mathscr{V}_{S}$ is the Regge-Wheeler potential for scalar perturbations. This equation was originally derived in [10] employing the Newman-Penrose formalism. Notice the striking similarity between the ReggeWheeler potential for the massless scalar field perturbations, $\mathscr{V}_{S}$, and the potential for gravitational perturbations, $\mathscr{V}_{T}[13]$.

The electromagnetic case is even simpler. For this case, the covariant ReggeWheeler equation (82) for the electric field perturbation $\mathscr{E}, \mathscr{E}=r^{-2} E$, reduces in the Schwarzschild background to

$$
\ddot{E}-\hat{\hat{E}}-\mathcal{A} \hat{E}-\delta^{2} E=0 \text {. }
$$

Adopting once more scalar spherical harmonics and proceeding as before leads to

$$
\left(-\frac{\partial^{2}}{\partial t^{2}}+\frac{\partial^{2}}{\partial r_{*}^{2}}+\mathscr{V}_{V}\right) E_{\mathrm{S}}=0, \quad \mathscr{V}_{V}=\left(1-\frac{2 m}{r}\right)\left[\frac{L}{r^{2}}\right],
$$

where $\mathscr{V}_{V}$ is the Regge-Wheeler potential for electromagnetic perturbations (the magnetic case is completely analogous). The Regge-Wheeler equation for radial electric perturbations (110), and its magnetic counterpart, was originally derived in [11] employing the Newman-Penrose formalism.

It is remarkable that the (source-free) electromagnetic perturbations can also be described by a linear first order system of ODE's after expanding Maxwell's equations into spherical and time harmonics [2] (possible because the background is static). The time derivatives of first order quantities are decomposed into their Fourier components by assuming an $e^{i \omega \tau}$ time dependence for the first order variables; factors of $i \omega$ just represent time derivatives, $d / d \tau$. Note that

$$
\hat{\omega}=-\mathcal{A} \omega \Rightarrow \omega=\sigma\left(1-\frac{2 m}{r}\right)^{-1 / 2}=\frac{2 \sigma}{\phi r},
$$

arising from the commutation relation between the dot- and hat-derivatives. The harmonic function $\omega$ is defined with respect to proper time $\tau$ of observers moving along $u^{a}$, while $\sigma$ is the constant harmonic index associated with time $t$ of observers at infinity. They are related by $\omega \tau=\sigma t$. Inserting time harmonics into equations (86) - (91) one finds that equations (88)- (89) turn into constraints for the odd modes $\overline{\mathscr{E}} \mathrm{V}$ and $\overline{\mathscr{B}}_{\mathrm{V}}$, respectively,

$$
\overline{\mathscr{B}} \mathrm{V}=\frac{i \omega r}{L} \mathscr{E} \mathrm{S}, \quad \overline{\mathscr{E}}_{\mathrm{V}}=-\frac{i \omega r}{L} \mathscr{B}_{\mathrm{S}}
$$


which may subsequently be eliminated from equations (90)-(91). The final result is a linear first order system of ODE's,

$$
\hat{\mathbf{Y}}=\mathbf{A Y},
$$

where the matrix $\mathbf{A}$ is given as

$$
\mathbf{A}=\left(\begin{array}{cc}
-\phi & \frac{L}{r} \\
\frac{1}{r}-\frac{r \omega^{2}}{L} & -\frac{1}{2} \phi-\mathcal{A}
\end{array}\right)
$$

and the perturbation vector $\mathbf{Y}$ is defined in the case of polar and axial perturbations by

$$
\mathbf{Y}_{\text {polar }}=\left(\begin{array}{c}
\mathscr{E} S \\
\mathscr{E} \mathrm{V}
\end{array}\right) \quad \text { and } \quad \mathbf{Y}_{\text {axial }}=\left(\begin{array}{c}
\mathscr{B} \mathrm{S} \\
\mathscr{B} \mathrm{V}
\end{array}\right)
$$

respectively. We emphasise the observation that in a stationary situation, e.g. $\omega=0$, the constraints (112) imply $\overline{\mathscr{E} V}=\overline{\mathscr{B}}_{V}=0$ in the absence of charges, which means that electromagnetic perturbations cannot have a solenoidal part in the sheet. A further implication is that a pure magnetic field can only exist on the Schwarzschild geometry if it has no solenoidal contribution in the sheet and is stationary and/or sheet-like. In the stationary case, the system (113) can be solved analytically. For example, picking $L=2$ in (114) easily yields the well-known solutions of a static magnetic dipole or a static uniform magnetic field at infinity (compare also [26-28]).

\subsection{Vaidya Spacetime}

5.2.1. The background The energy-momentum tensor of Vaidya's radiating sphere spacetime is that of a radiation fluid, e.g.,

$$
T_{a b}=\mu k_{a} k_{b}, \quad k_{a} k^{a}=0 .
$$

A convenient form of its metric for the case of outgoing radiation is given in terms of retarded time $u=t-r_{*}$ by

$$
g_{a b}=-\left(1-\frac{2 m(u)}{r}\right) d u^{2}-2 d u d r+r^{2} d \Omega^{2},
$$

where $m(u)$ is a arbitrary non-increasing function, $m^{\prime}(u) \equiv d m(u) / d u \leq 0$; if $m(u)$ is constant, the metric is equivalent to the Schwarzschild metric. Thus, the Vaidya spacetime describes a collapsing star that irradiates a part of its mass, where the total power output measured at infinity is given by $-m^{\prime}(u)=4 \pi r^{2} \mu[29-34]$.

A covariant description of the Vaidya spacetime is obtained as follows. Spherical symmetry requires the null-vector $k_{a}$ to be of the form $k_{a}=u_{a} \pm n_{a}$, where a plus (minus) corresponds to outflowing (inflowing) radiation. Thus, a static observer will encounter the radiation fluid as an imperfect one, with the energy-density $\mu$, the isotropic pressure $p=\frac{1}{3} \mu$, the radial heat-flux $Q= \pm \mu$ and the trace-free part of the anisotropic sheet pressure $\Pi=\frac{2}{3} \mu$. Working in the static frame $(\dot{r}=0)$, the nonstationary spherically symmetric Vaidya spacetime in the case of outgoing radiation is described covariantly by the following set of equations:

$$
\begin{aligned}
\hat{\mathcal{A}}-\dot{\theta} & =-\mathcal{A}(\mathcal{A}+\phi)+\theta(\theta-\phi), \\
\hat{\phi} & =-\phi\left(\frac{1}{2} \phi-\mathcal{A}-2 \theta\right), \\
\dot{\phi} & =-\theta \phi, \\
\hat{\theta}+\dot{\theta} & =-\theta\left(\frac{1}{2} \phi+3 \theta+3 \mathcal{A}\right) .
\end{aligned}
$$


The corresponding constraints, implied by our frame choice, are

$$
\begin{aligned}
K & \equiv r^{-2}=\frac{1}{4} \phi^{2}-\mathcal{E}, \\
\Sigma & =\frac{2}{3} \theta, \\
\mu & =-\theta \phi=3 p=Q=\frac{3}{2} \Pi, \\
\mathcal{E} & =\mu-\mathcal{A} \phi .
\end{aligned}
$$

Note that the equations governing the Vaidya spacetime reduce to the ones describing Schwarzschild spacetime [see (96)-98] ] when the expansion $\theta$ vanishes.

In order to solve the equations, we find it useful to express our derivatives in terms of coordinates $\{r, u\}$, where $r$ is the radial parameter and $u$ labels time. In particular, we have $\rho=\rho(r, u)$ and $\tau=\tau(r, u)$ and the derivatives become for an arbitrary scalar $X$ :

$$
\begin{aligned}
& \hat{X}=\frac{r \phi}{2} \frac{\partial X}{\partial r}+\hat{u} \frac{\partial X}{\partial u}, \\
& \dot{X}=\dot{u} \frac{\partial X}{\partial u} .
\end{aligned}
$$

One neat possibility to define the coordinate $u$ is through the relations

$$
\dot{u}=-\hat{u}=\frac{1}{\hat{r}}=\frac{2}{r \phi},
$$

which is compatible with the commutator relation (45) and identifies $u$ with the $u$ coordinate used in the metric (117), as will become clear in an instant. Upon switching to the coordinates $\{r, u\}$ and putting $\mathcal{A} \equiv \mathcal{A}_{\mathrm{Sch}}-\theta$ [as suggested by equations (119) and [121] ], the system of equations transforms into

$$
\begin{aligned}
\left(\frac{1}{4} r^{2} \phi^{2} \partial_{r}-\partial_{u}\right) \mathcal{A}_{\mathrm{Sch}} & =-\frac{1}{2} r \phi\left[\mathcal{A}_{\mathrm{Sch}}\left(\mathcal{A}_{\mathrm{Sch}}+\phi+\theta\right)+\frac{1}{2} \phi \theta\right], \\
r \partial_{r} \phi & =-\phi+2 \mathcal{A}_{\mathrm{sch}}, \\
2 \partial_{u} \phi & =-r \theta \phi^{2}, \\
r \phi \partial_{r} \theta & =-\theta\left(\phi+6 \mathcal{A}_{\mathrm{Sch}}\right) .
\end{aligned}
$$

The solutions may be readily found by making an educated guess. Clearly, by spherical symmetry, the radiation has to move outward radially and the expansion $\phi$ of the radial congruence $n^{a}$ therefore should be of the same form as for the Schwarzschild spacetime but with a decreasing mass parameter. Since equation (130) is identical with the analogous one for the Schwarzschild spacetime, we are led to identify $\mathcal{A}_{\text {sch }}$ with the acceleration of a static Schwarzschild observer, in particular, $\mathcal{A}_{\text {sch }}$ represents the acceleration caused by the star's instantaneous gravitational field. It is then straightforward to work out all other quantities. The result is:

$$
\begin{aligned}
& \phi=\frac{2}{r} \sqrt{1-\frac{2 m(u)}{r}} \\
& \theta=\frac{m^{\prime}(u)}{r}\left(1-\frac{2 m(u)}{r}\right)^{-3 / 2}=\frac{3}{2} \Sigma \\
& \mathcal{A}=\mathcal{A}_{\text {Sch }}-\theta ; \quad \mathcal{A}_{\mathrm{Sch}}=\frac{m(u)}{r^{2}}\left(1-\frac{2 m(u)}{r}\right)^{-1 / 2}, \\
& \mu=-\frac{2 m^{\prime}(u)}{r^{2}}\left(1-\frac{2 m(u)}{r}\right)^{-1}=3 p=Q=\frac{3}{2} \Pi, \\
& \mathcal{E}=-\frac{2 m(u)}{r^{3}}
\end{aligned}
$$


It is worth pointing out that these solutions can also be worked out directly from the Vaidya metric (117) by choosing the splitting unit vectors to be

$$
u^{a}=\left(1-\frac{2 m(u)}{r}\right)^{-1 / 2}\left(\frac{\partial}{\partial u}\right)^{a}
$$

and

$$
n^{a}=-\left(1-\frac{2 m(u)}{r}\right)^{-1 / 2}\left(\frac{\partial}{\partial u}\right)^{a}+\left(1-\frac{2 m(u)}{r}\right)^{1 / 2}\left(\frac{\partial}{\partial r}\right)^{a}
$$

respectively.

The obtained solutions may be interpreted as follows. First note that from equation (136) follows that a physically acceptable, that is, positive energy density $\mu$ requires a non-increasing (but otherwise unrestricted) mass, $m^{\prime}(u) \leq 0$, suggesting that a part of the star's mass is released as radiation during collapse, with total luminosity $-m^{\prime}(u)=4 \pi r^{2} \mu$ at infinity (in proper units). Further, the expansion $\theta$ and shear $\Sigma$ are negative since $m^{\prime}(u) \leq 0$, meaning that the spatial sections of the spacetime are contracting, which tends to push nearby observers together. Equation (135) hence tells us that an observer has to balance its always radially outward directed acceleration according to the diminishing gravitational attraction of the collapsing star and the growing contraction in order to stay at rest. Finally, the expressions for sheet expansion $\phi$ and tidal forces $\mathcal{E}$ are as for a star in equilibrium but with a time-dependent mass due to mass-radiation conversion during collapse.

5.2.2. The perturbations With the background solutions for the Vaidya spacetime at hand, it is now an easy task to rewrite the covariant Regge-Wheeler equations for the case of scalar and electromagnetic perturbations into in terms of coordinates. From equation (68) one obtains immediately for a massive scalar field $\psi$ with mass $M, \psi=r^{-1} \Psi$, that

$$
\ddot{\Psi}-\hat{\hat{\Psi}}-\mathcal{A} \hat{\Psi}+\theta \dot{\Psi}-\left[\mathcal{E}-M^{2}+\delta^{2}\right] \Psi=0,
$$

while equation (82) for the electromagnetic perturbations, $\mathscr{E}=r^{-2} E$, gives

$$
\ddot{E}-\hat{\hat{E}}-\mathcal{A} \hat{E}+\theta \dot{E}-\delta^{2} E=0,
$$

Introducing scalar spherical harmonics and adopting the coordinates $\{r, u\}$ from above, we find the corresponding Regge-Wheeler equation

$$
\left[\left(1-\frac{2 m(u)}{r}\right) \frac{\partial^{2}}{\partial r^{2}}-2 \frac{\partial^{2}}{\partial u \partial r}+\frac{2 m(u)}{r^{2}} \frac{\partial}{\partial r}+\left(\frac{L}{r^{2}}+\lambda \frac{2 m(u)}{r^{3}}+\lambda M^{2}\right)\right] \mathscr{P}_{\mathrm{S}}=0
$$

where $\mathscr{P}_{\mathrm{S}}$ denotes the scalar harmonic component of either $\Psi$ or $E$ and $L=\ell(\ell+1)$; the parameter $\lambda$ takes the value one for perturbations in a scalar field and the value zero for electromagnetic perturbations.

It is instructive to compare the equations (142) with the corresponding equations (108) and (110) in the Schwarzschild case. We found it most favourable to do the comparison by employing coordinates $\left\{t, r_{*}\right\}$ which are defined for $r>2 m(u)$ by

$$
t=u+r_{*}, \quad r_{*}=r+2 m(u)\left[\ln \left(\frac{r}{2 m(u)}-1\right)\right] .
$$


In terms of the coordinates $\left\{t, r_{*}\right\}$, the equations (142) now become

$$
\begin{gathered}
\{[-1-h(u, r)] \\
\frac{\partial^{2}}{\partial t^{2}}+[1-h(u, r)] \frac{\partial^{2}}{\partial r_{*}^{2}}-\frac{4 m^{\prime}(u)}{r-2 m(u)}\left(\frac{\partial}{\partial t}+\frac{\partial}{\partial r_{*}}\right)-2 h(u, r) \frac{\partial^{2}}{\partial t \partial r_{*}} \\
\left.+\left(1-\frac{2 m(u)}{r}\right)\left[\frac{L}{r^{2}}+\lambda \frac{2 m(u)}{r^{3}}+\lambda M^{2}\right]\right\} \mathscr{P}_{\mathrm{S}}=0
\end{gathered}
$$

where the function $h(u, r)$,

$$
h(u, r)=4 m^{\prime}(u)\left[\ln \left(\frac{r}{2 m(u)}-1\right)-\left(1-\frac{2 m(u)}{r}\right)^{-1}\right],
$$

was introduced for brevity's sake. Clearly, when the mass $m(u)$ stays constant, the equations (144) reduce to the familiar Regge-Wheeler equations (108) and (110), respectively. Note that all new terms in the Regge-Wheeler equations for the Vaidya spacetime (144) are proportional to the mass change in time, $m^{\prime}(u)$, and thus proportional to the expansion $\theta$ [cf equation (134)]. This was to be expected as, contrasting the Schwarzschild case, the only new ingredient in the covariant ReggeWheeler equations for the Vaidya spacetime [see (140) and (141)] is proportional to the expansion $\theta$ as well. Finally, the accompanying Regge-Wheeler potentials retain the Schwarzschild form but become time-dependent in the Vaidya case.

\subsection{More general spacetimes}

It was shown in [4] that the metric of every LRS class II spacetime can be given in diagonal form if local comoving coordinates are chosen:

$d s^{2}=-A^{-2}(t, x) d t^{2}+B^{2}(t, x) d x^{2}+C^{2}(t, x)\left[d y^{2}+D^{2}(y, k) d z^{2}\right]$,

where $D(y, k)=(\sin y, y, \sinh y)$ for $k=(1,0,-1)$ labelling the closed, flat or open geometry of the sheet. The kinematical quantities are now

$$
\begin{aligned}
\phi & =2 \frac{\hat{C}}{C}, \\
\mathcal{A} & =-\frac{\hat{A}}{A}, \\
\theta & =\frac{\dot{B}}{B}+2 \frac{\dot{C}}{C}, \\
\Sigma & =\frac{2}{3}\left(\frac{\dot{B}}{B}-\frac{\dot{C}}{C}\right),
\end{aligned}
$$

and equations (39) and (40) can therefore be integrated to yield the Gaussian curvature

$$
K=\frac{C_{1}}{C^{2}(t, x)}
$$

where the integration constant $C_{1}$ may be normalised such that $C_{1}=k$. From equation (26) or (28) one immediately obtains for the heatflux

$$
Q=2\left(\frac{\hat{\dot{C}}}{C}-\frac{\dot{B} \hat{C}}{B C}\right)
$$


while equation (31) directly yields the relation

$$
\frac{1}{2}(\mu+3 p-2 \Lambda)=-\left[\frac{\hat{\hat{A}}}{A}-2\left(\frac{\hat{A}}{A}\right)^{2}+\frac{\ddot{B}}{B}+2 \frac{\ddot{C}}{C}+2 \frac{\hat{A} \hat{C}}{A C}\right],
$$

which can be employed to constrain the metric functions such that, e.g., $\mu+3 p>0$ holds. If a simple equation of state, $p=\alpha \mu$, say, is assumed, one finds from this relation an expression for the energy density $\mu$. On the other hand, the field equations imply

$$
\mu+\Lambda-K=\left(\frac{\dot{C}}{C}\right)^{2}-\left(\frac{\hat{C}}{C}\right)^{2}+2 \frac{\dot{B} \dot{C}}{B C}-2 \frac{\hat{C}}{C}
$$

which is in general different from the expression for $\mu$ obtained from equation (153) and therefore gives an additional constraint for the metric functions. Finally, the pressure variables and the electric Weyl curvature can be calculated similarly but the resulting expressions are somewhat long and will not be stated here.

5.3.1. The perturbations When local comoving coordinates are chosen, the wave equations governing the scalar and electromagnetic perturbations in the sourcefree case are best given in form of equation (67) and (82), respectively, since there only the kinematical variables enter and these are simple expressions in terms of the metric functions [cf. equations (147)-(150)]. The corresponding wave equations read in the case of scalar field perturbations $\psi$ as

$$
\ddot{\psi}-\hat{\hat{\psi}}+\left(\frac{\dot{B}}{B}+2 \frac{\dot{C}}{C}\right) \dot{\psi}+\left(\frac{\hat{A}}{A}-2 \frac{\hat{C}}{C}\right) \hat{\psi}+\left(M^{2}-\delta^{2}\right) \psi=0,
$$

while in the case of (rescaled) electromagnetic perturbations $E$ (or $B$ ) they become

$$
\ddot{E}-\hat{\hat{E}}+\left(\frac{\dot{B}}{B}\right) \dot{E}+\left(\frac{\hat{A}}{A}\right) \hat{E}+\left[3 \frac{\dot{B} \dot{C}}{B C}-2\left(\frac{\dot{C}}{C}\right)^{2}-\delta^{2}\right] E=0 .
$$

Once the metric functions $A, B$ and $C$ in (146) are given for a known LRS class II solution, these wave equations may readily be transformed into their concomitant coordinate analogues.

Observe that the 'radial' parameter $r$, covariantly defined by the relations (54), agrees with the metric function $C(t, x)$ in the line element (146) in account of (147) and (149)-(150). Thus the physical electromagnetic perturbations $\mathscr{E}$ are obtained from the rescaled ones $E$ by the transformation $\mathscr{E}=C^{-2}(t, x) E$. If the scalar field $\psi$ is analogously rescaled as $\psi=C^{-1}(t, x) \Psi$, then the wave equation for the scalar field perturbation (155) takes the Regge-Wheeler form

$\ddot{\Psi}-\hat{\hat{\Psi}}+\left(\frac{\dot{B}}{B}\right) \dot{\Psi}+\left(\frac{\hat{A}}{A}\right) \hat{\Psi}+\left[M^{2}+\frac{\hat{\hat{C}}}{C}-\frac{\dot{C}}{C}-\frac{\dot{B} \dot{C}}{B C}-\frac{\hat{A} \hat{C}}{A C}-\delta^{2}\right] \Psi=0$.

It is thus obvious that once a harmonic decomposition has been applied to the equations (156) and (157), the resulting wave equations will only differ in their corresponding potential term. 
If in addition to the LRS symmetry further symmetries are present, then the wave equations for the perturbations simplify considerably. For example, for stationary LRS class II spacetimes we have

$$
\begin{aligned}
& \ddot{\Psi}-\hat{\hat{\Psi}}+\left(\frac{\hat{A}}{A}\right) \hat{\Psi}+\left[M^{2}+\frac{\hat{\hat{C}}}{C}-\frac{\hat{A} \hat{C}}{A C}-\delta^{2}\right] \Psi=0, \\
& \ddot{E}-\hat{\hat{E}}+\left(\frac{\hat{A}}{A}\right) \hat{E}-\delta^{2} E=0
\end{aligned}
$$

while for spatially homogeneous spacetimes (where one may set $A=1$ ) we have

$$
\begin{aligned}
& \ddot{\Psi}-\hat{\hat{\Psi}}+\left(\frac{\dot{B}}{B}\right) \dot{\Psi}+\left[M^{2}-\frac{\dot{C}}{C}-\frac{\dot{B} \dot{C}}{B C}-\delta^{2}\right] \Psi=0, \\
& \ddot{E}-\hat{\hat{E}}+\left(\frac{\dot{B}}{B}\right) \dot{E}+\left[3 \frac{\dot{B} \dot{C}}{B C}-2\left(\frac{\dot{C}}{C}\right)^{2}-\delta^{2}\right] E=0 .
\end{aligned}
$$

5.3.2. Application - LTB dust Universe As an example of an inhomogeneous spacetime, let us consider the Lemaitre-Tolman-Bondi (LTB) [15,17-19] dust Universe (with $\Lambda=0$ ), whose metric is given by

$$
d s^{2}=-d t^{2}+\frac{\left(Y^{\prime}\right)^{2}}{k-\varepsilon f^{2}(r)} d r^{2}+Y^{2}\left[d \vartheta^{2}+D^{2}(\vartheta, k) d \varphi^{2}\right]
$$

Here, a prime means $\partial / \partial r$ and a dot will denote $\partial / \partial t$. Moreover, $Y=Y(t, r)$ is the solution of $\dot{Y}^{2}-2 m(r) / Y=-\varepsilon f^{2}(r)$, wherein $\varepsilon=(-1,0,1)$ corresponds to the hyperbolic, parabolic and elliptic solution, respectively. The constraint equation can be integrated completely, which yields an additional function $t_{B}(r)$. Therefore, there are three functions which can be prescribed at will: the 'mass' $m(r)$, the 'energy' $f(r)$ and the 'bang time' $t_{B}(r)$.

It follows from equations (147)-1150) that the acceleration $\mathcal{A}$ has to vanish and that the non-zero dynamical variables take the form

$$
\begin{aligned}
\phi & =2 \frac{\sqrt{k-\varepsilon f^{2}}}{Y}, \\
\theta & =\frac{\dot{Y}^{\prime}}{Y^{\prime}}+2 \frac{\dot{Y}}{Y}, \\
\Sigma & =\frac{2}{3}\left(\frac{\dot{Y}^{\prime}}{Y^{\prime}}-\frac{\dot{Y}}{Y}\right) .
\end{aligned}
$$

An expression for the energy density $\mu$ can be gained from equation (153) and yields, using the above mentioned constraint,

$$
\mu=\frac{2 m^{\prime}}{Y^{\prime} Y^{2}} \text {. }
$$

Finally, the wave equations (156) and (157) governing the perturbations $\mathscr{P}$ can be written as

$$
\ddot{\mathscr{P}}-\frac{k-\varepsilon f^{2}}{\left(Y^{\prime}\right)^{2}}\left[\mathscr{P}^{\prime \prime}-\left(\frac{Y^{\prime \prime}}{Y^{\prime}}+\frac{\varepsilon f f^{\prime}}{k-\varepsilon f^{2}}\right) \mathscr{P}^{\prime}\right]+\frac{\dot{Y}^{\prime}}{Y^{\prime}} \dot{\mathscr{P}}+\left(V-\delta^{2}\right) \mathscr{P}=0 ;
$$


the potential $V$ is given by

$$
V=V_{E M}=3 \frac{\dot{Y} \dot{Y}^{\prime}}{Y Y^{\prime}}-2\left(\frac{\dot{Y}}{Y}\right)^{2}
$$

in the case of electromagnetic perturbations, whereas

$$
V=V_{S}=M^{2}+\frac{m}{Y^{3}}+\left(\frac{\dot{Y} \dot{Y}^{\prime}+\varepsilon f f^{\prime}}{Y Y^{\prime}}\right)
$$

denotes the potential in the case of scalar field perturbations.

5.3.3. Application - Kantowski-Sachs dust Universe As a further example we consider a spherically symmetric Kantowski-Sachs dust Universe with $\Lambda=0[15,20,21]$. The metric writes as

$$
d s^{2}=-d t^{2}+B^{2}(t) d r^{2}+C^{2}(t) d \Omega^{2},
$$

where the metric functions are conveniently expressed in terms of a parameter $\eta(t)$ satisfying $d t=2 C d \eta$, namely

$$
\begin{aligned}
& B=m(\eta \tan \eta+1)+b \tan \eta, \\
& C=c \cos ^{2} \eta ; \quad m, b, c=\text { const } .
\end{aligned}
$$

Since this spacetime is spatially homogeneous, the acceleration $\mathcal{A}$ and expansion $\phi$ of the fundamental observer's congruence have to vanish [cf. equations (148)-(147)]. The remaining quantities are found to be

$$
\begin{aligned}
\mu & =\frac{m}{B C^{2}}, \\
\mathcal{E} & =\frac{m \cos ^{2} \eta-3 B}{3 B C^{2} \cos ^{2} \eta}, \\
\theta & =c \frac{m\left(\eta-3 \sin \eta \cos \eta-4 \eta \sin ^{2} \eta\right)+b\left(1-4 \sin ^{2} \eta\right)}{2 B C^{2}}, \\
\Sigma & =c \frac{m\left(\eta+3 \sin \eta \cos \eta+2 \eta \sin ^{2} \eta\right)+b\left(1+2 \sin ^{2} \eta\right)}{3 B C^{2}} .
\end{aligned}
$$

The equations for the perturbations $\mathscr{P}$ now become in terms of the parameter $\eta$

$$
\left\{\frac{\partial^{2}}{\partial \eta^{2}}-\frac{4 C^{2}}{B^{2}} \frac{\partial^{2}}{\partial r^{2}}+\frac{B\left(1+2 \sin ^{2} \eta\right)-m \cos ^{2} \eta}{B \sin \eta \cos \eta} \frac{\partial}{\partial \eta}+4 C^{2}\left(V-\delta^{2}\right)\right\} \mathscr{P}=0,
$$

where

$$
V=V_{S}=M^{2}+\frac{2 B-m \cos ^{2} \eta}{2 B C^{2} \cos ^{2} \eta}
$$

in the case of scalar perturbations, and

$$
V=V_{E M}=\frac{3 m \cos ^{2} \eta-B\left(3+4 \sin ^{2} \eta\right)}{2 B C^{2} \cos ^{2} \eta}
$$

in the case of electromagnetic perturbations, respectively. 
Scalar field and electromagnetic perturbations on LRS spacetimes

\section{Conclusion}

Employing the $1+1+2$ formalism of Clarkson \& Barrett [2], we presented a covariant description of LRS class II spacetimes in terms of scalar quantities, which all have either a clear physical or geometrical meaning. We investigated scalar and electromagnetic perturbations (test fields) on LRS class II spacetimes and found that they are governed by covariant wave equations [see equations [68) and [82)], which are the covariant generalisations of the Regge-Wheeler equation, known to describe perturbations of the Schwarzschild spacetime. In particular, it was shown that both scalar and electromagnetic perturbations (in the absence of sources) are governed by master equations of the same form, namely the covariant generalised Regge-Wheeler equation,

$$
\ddot{\mathscr{P}}-\hat{\hat{\mathscr{P}}}-\mathcal{A} \hat{\mathscr{P}}+\left(\Sigma+\frac{1}{3} \theta\right) \dot{\mathscr{P}}+\left(V-\delta^{2}\right) \mathscr{P}=0,
$$

where the potential $V$ is different for the two cases considered. To arrive at this specific form, one had to rescale the perturbations with the 'radial' parameter $r$, which is induced from the Gaussian curvature $K$ of the sheet and can thus only be defined covariantly for LRS class II spacetimes [cf (54)]. The findings have been discussed in some detail for the particular cases of Schwarzschild and Vaidya spacetimes, and for some dust Universe models. While the master equations may be written simply as equation (180) in covariant form, they can become very untidy when written explicitly in coordinates, demonstrating some of the advantages of using a covariant approach.

\section{Acknowledgements}

It is a pleasure to thank Mattias Marklund and Peter Dunsby for their continuous advice and encouragement on this project, as well as George Ellis and Charles Hellaby for discussions.

\section{References}

[1] Ellis G F R and van Elst H 1999 in Theoretical and Observational Cosmology, pp. 1-116 ed. Marc Lachièze-Rey (Kluwer, Dordrecht)

[2] Clarkson C A and Barrett R 2003 Class. Quantum Grav. 203855

[3] Ellis G F R 1967 J. Math. Phys. 81171

[4] Stewart J M and Ellis G F R 1968 J. Math. Phys. 91072

[5] van Elst H and Ellis G F R 1996 Class. Quantum Grav. 131099

[6] Marklund M 1997 Class. Quantum Grav. 141267

[7] Marklund M and Bradley M 1999 Class. Quantum Grav. 161577

[8] Mustapha N, Ellis G F R, van Elst H and Marklund M 2000 Class. Quantum Grav. 173135

[9] Regge T and Wheeler J A 1957 Phys. Rev. 1081063

[10] Price R H 1972 Phys. Rev. D 52419

[11] Price R H 1972 Phys. Rev. D 52439

[12] Chandrasekhar S 1983 The Mathematical Theory of Black Holes, (Clarendon Press, Oxford)

[13] Nollert H-P 1999 Class. Quantum Grav. 16 R159-R216

[14] Kokkotas K D and Schmidt B G 1999 Living Rev. Relativity 22 , http//www . livingreviews.org/Articles/Volume2/1999-2kokkotas/

[15] Kramer D, Stephani H, MacCallum M and Herlt E 1980 Exact solutions of Einstein's field equations (Cambridge University Press)

[16] Vaidya P C 1951 Proc. Indian Acad. Sci. A33 264

[17] Lemaitre G 1933 Ann. Soc. Sci. Bruxelles I A 5351 Translation: Lemaitre G 1997 Gen. Rel. Grav. 29641

[18] Tolman R C 1934 Proc. Nat. Acad. Sci. U.S. 2069

[19] Bondi H 1947 Mon. Not. Roy. Ast. Soc. 107410 
[20] Kompaneets A S and Chernov A S 1965 Sov. Phys.-JETP 201303

[21] Kantowski R and Sachs R K 1966 J. Math. Phys. 7443

[22] Tsamparlis M and Mason D P 1983 J. Math. Phys. 241577

[23] Stewart J M and Walker M 1974 Proc. R. Soc. London A 34149

[24] Dunsby P K S, Bruni M and Ellis G F R 1992 ApJ 39557

[25] Clarkson C A, Marklund M, Betschart G and Dunsby P K S 2004 Astrophys. J. 613492

[26] Wald R M 1974 Phys. Rev. D 101680

[27] Hanni R S and Ruffini R 1976 Lett. Nuovo Cimento 15189

[28] Sonego S and Abramowicz M A 1998 J. Math. Phys. 393158

[29] Vaidya P C 1952 Curr. Science 2196

[30] Vaidya P C 1953 Nature 171260

[31] Raychaudhuri A K 1953 Z. Physik 135225

[32] Israel W 1958 Proc. Roy. Soc. (London) A248 404

[33] Lindquist R W, Schwartz R A and Misner C W 1965 Phys. Rev. 1371364

[34] Poisson E and Israel W 1990 Phys. Rev. D 411796 